\title{
Beam Steering for Circular Switched Parasitic Arrays using a Combinational Approach
}

\author{
Mofolo R. O. Mofolo ${ }^{1}$, Albert A. Lysko, Thomas O. Olwal \\ Wireless Computing \& Networking Research Group \\ CSIR Meraka Institute \\ Pretoria, South Africa \\ \{mmofolo,alysko, tolwal\}@,csir.co.za
}

\author{
Willem A. Clarke \\ School of Electrical Engineering \\ University of Johannesburg ${ }^{1}$ \\ Johannesburg, South Africa \\ willemc@uj.ac.za
}

\begin{abstract}
In this paper we present a method of electronic beam steering for circular switched parasitic array (SPA) antennas. In circular SPA antennas, one achieves azimuth beam steering by open-circuiting and short-circuiting different parasitic elements, usually with only one parasitic element open-circuited at a time. For the SPA antenna with few parasitic elements, this results in low azimuth beam steering resolution. In the proposed method, we iterate through different combinations of parasitic elements and the possible switch states of the lumped impedance loads connected to the parasitic elements. Our method aims to increase the azimuth beam steering resolution of the circular SPA antennas. The method is verified using a combination of simulation (using both MATLAB and WIPL-D) and a SPA antenna prototype implementation. The MATLAB code uses the induced EMF method, while the WIPL-D uses the Methods of Moment (MoM) for solving the antenna impedances. The three sets of results (simulations and measurement) match very well at 2.4 GHz. The results indicate the availability of more options (different loading configurations) for electronic beam switching that can be adopted to improve the beam steering resolution of circular SPA antennas.
\end{abstract}

Keywords-Antenna radiation patterns; circular switched parasitic arrays; electronic beam steering; method of moments; induced EMF method.

\section{INTRODUCTION}

Smart antennas are recommended for the performance improvements in throughput, capacity and coverage of the wireless networks [1-3]. However, the usual smart antennas involve complex signal processing and hence result in a power hungry system. Also, each array element is connected to the feed circuitry and requires impedance matching and phase control. These factors result in increased power consumption, complexity, the size of the array and overall system cost. They make smart antenna systems unsuitable and unaffordable for some applications, especially considering network deployment in African rural areas where most network devices are battery powered.

To take advantage of the performance improvements offered by the smart antennas, an alternative antenna system is considered. The proposed antenna system is formulated using parasitic arrays, and employs aerial beamforming (ABF) [4-6]. In $\mathrm{ABF}$, only one active element needs to be connected to the RF port. The other elements are parasitic without connection to the RF port. The active element radiates fields that induce currents in the parasitic elements, and the parasitic elements reradiate it [5]. This interaction between antenna elements is due to mutual coupling, which is a fundamental for the functioning and operation of the parasitic antenna arrays.

For systems using ABF, only one amplifier and impedance matching circuitry need to be designed. Parasitic arrays are capable of beam steering which is realized by electronically controlling the lumped impedance loads connected to the bodies of the parasitic elements [4], [7], [8]. An appropriate control of impedance loads connected to the bodies of parasitic elements enables these elements to be switched between the reflector and director states as in the Yagi-Uda arrays [7], [9].

Switched parasitic array (SPA) antenna beam steering is realized by a proper choice of combination of parasitic elements to act as directors and reflectors [7]. Beam steering using parasitic arrays could be achieved over both planes: in the elevation and azimuth [10].

Generally, depending on the type of impedance loads connected to the bodies of the parasitic elements, parasitic arrays can assume adaptive or switched configuration. An adaptive configuration is realized by connecting reactive (capacitive and/or inductive) loads [11]. Alternatively, a switched beam configuration can be realized by connecting switchable (usually resistive) loads to the bodies of parasitic elements and are named switched parasitic arrays (SPA) antenna [4], [12-15].

The proposed beam steering method for the circular SPA antennas is presented. This method is aimed at increasing the azimuth beam steering resolution of the SPA antennas. The advantage of this proposed method is that three more parasitic loading configurations have been realized. These configurations can be used for the beam steering of the SPA antennas, as compared to only one traditional loading configuration. For instance, the loading configuration of opencircuiting two neighboring parasitic elements, which yields best result in this paper, has not been published to the best of our knowledge.

\section{RELATED WORK}

The concept of beam steering using parasitic radiators by electronically controlling tunable loads connected to them was

This work was supported by the CSIR Meraka Institute and the University of Johannesburg, South Africa. 
introduced by Harrington [16] in the 1970's. This beam steering technique was adopted and simplified by Milne [14] in early 1980's. Milne replaced the tunable loads with a simple On/Off RF switch (using PIN diodes). Significant research work has been conducted up to date on the SPA antennas with focus on using dipole, monopoles and microstrip patch antennas as the parasitic elements to form different array sizes [14], [15].

Usually, regardless of the number and type of the array elements, the beam steering for the SPA antennas is performed using the following approach. The beam steering for a single ring circular SPA antenna such as the one shown in Fig. 1, is achieved by open-circuiting (isolating) one parasitic element while the other parasitic elements are short-circuited [4], [14], [15], [17]. The open-circuited parasitic elements act as directors, and the short-circuited parasitic elements function as reflectors like in the Yagi-Uda antennas.

In [4], [14], [15], [17], different array sizes and antenna elements have been used to design circular SPA antennas. In all cases, authors have used one beam steering approach of open-circuiting one parasitic element at a time. The main beam (radiation pattern with maximum gain) of the SPA antennas is directed towards the open-circuited parasitic element which is acting as a director. Therefore, there are $P$ (where $P$ is the number of parasitic elements) number of directions in which the main beam is directed. Normally, only one direction out of the $P$ directions needs to be optimized because of the array symmetry advantage [12]. This beam steering approach produces limited beam steering resolution.

The On/Off RF switches are mostly used in the design of SPA antennas [14] to electronically switch the parasitic elements between the director and reflector states. There are several technologies used in fabrication of such RF switches for instance, Micro-Electrical Mechanical Systems (MEMS) [18]. However, in this study, the parasitic elements are directly shorted to the ground plane and isolated from the ground plane using a thin sheet of insulation material.

\section{SYSTEM MODEL}

The system studied in this paper is a single ring circular switched parasitic array antenna with a total of five elements $(N=5)$ : one central active element surrounded by four parasitic elements $(P=4)$ as illustrated in Fig. 1.

The structural specifications of the SPA antenna consisting of dipole elements as shown in Fig. 1 are illustrated as follows:

- $\quad$ The length of active dipole element, $L_{a}=52 \mathrm{~mm}$;

- The length of all parasitic dipole elements, $L_{p}=56 \mathrm{~mm}$;

- The radius of the array dipole elements, $W r=0.8 \mathrm{~mm}$; and

- The placement of the parasitic elements from the centre, $d=62.5 \mathrm{~mm}$.

- $\quad$ Operational frequency, $f=2.4 \mathrm{GHz}$.

These structural parameters are estimated based on the array theory and most configurations used in the literature. All the

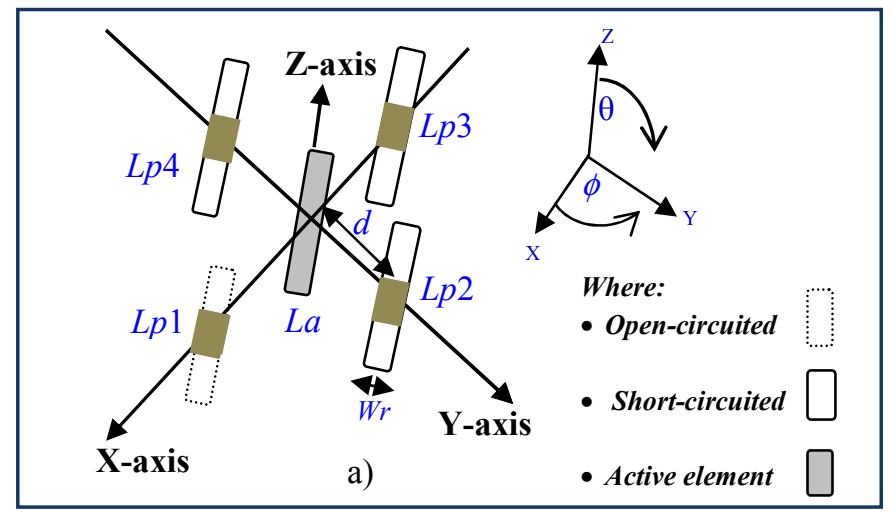

Fig. 1: Modelled antenna geometry with 5-Elements: 4 parasitic elements encircling 1 central active element.

parasitic elements are physically of the same length in order to achieve an omnidirectional radiation pattern, and maintain the symmetrical properties of the SPA antennas. Switching the parasitic elements between short-circuited and open-circuited states leads to variation in the elements' electrical length. An open-circuited or isolated parasitic element functions as a director because it becomes visually invisible as its electrical length shortens. Thus, parasitic elements can be toggled between functioning as directors and reflectors.

\section{PARASITIC ARRAY THEORY}

The array patterns are the result of the fields from all array elements constructively adding together in the desired directions and destructively cancelling each other in the remaining directions [14]. Constructive addition of the elements' fields in a specific direction yields directional radiation patterns with maximum gain. On the other hand, destructive cancellation of elements' fields in the remaining directions other than the desired direction produces the radiation pattern nulls. Radiation pattern nulls help in interference mitigation. The normalized far field radiation pattern for the SPA antenna such as the one in Fig. 1 is given by [19-21]:

$$
\begin{aligned}
& E_{\theta t}(\theta, \phi)=I_{0} \frac{\cos \left(k l_{0} \cos \theta\right)-\cos \left(k l_{0}\right)}{\sin \theta}+ \\
& \sum_{n=1}^{P}\left[I_{n} \frac{\cos \left(k l_{n} \cos \theta\right)-\cos \left(k l_{n}\right)}{\sin \theta} \times\right. \\
& \left.\mathrm{e}^{\left(j k d \sin \theta \cos \left(\phi-\phi_{n}\right)\right)}\right]
\end{aligned},
$$

where $k$ is the wave number. The symbols $I_{n}$ indicates the complex current (amplitude and phase) excitation of the $n^{\text {th }}$ element of the array respectively. $\phi_{n}$ represents the azimuth (phase) angle of $n^{\text {th }}$ parasitic element which is located at radius $d$ [19], [20]. $I_{0}$ represents the complex current excitation of the active element.

To estimate the performance of the SPA antennas such as the one shown in Fig. 1, firstly, analyses of the impedance matrix are carried out. The impedance matrix consists of the self and mutual impedances of array elements. Either an analytical technique such as induced electromotive force (EMF) method or numerical technique such as Method of Moments (MoM) can be used to compute the impedance matrix [22]. 
To obtain the currents in expression (1), an analysis of the system impedance matrix has to be performed. The impedance matrix can be obtained by determining the equivalent network [14], [19], corresponding to Fig. 1. The matrix presentation of the matrix form of the equivalent network equations can be represented [23] as:

$$
\mathbf{V}=\left[\mathbf{Z}+\mathbf{Z}_{\mathbf{L}}\right] \mathbf{I}
$$

where $\mathbf{V}$ is the column vector of voltage sources where only one entry ( $V_{l}=1$ volts) is non-zero. $\mathbf{I}$ is the column vector of the currents for all elements. $\mathbf{Z}$ is the square impedance matrix. If we assume all the parasitic elements are loaded with some impedance loads, $Z n_{L}$ (for $n=2,3,4,5$ ) across the terminals, the impedance loads across all the parasitic element terminals can be represented in a form of a diagonal matrix as [19], [20]:

$$
\mathbf{Z}_{\mathbf{L}}=\left[\begin{array}{ccccc}
0 & 0 & \cdots & 0 & 0 \\
0 & Z_{2_{L}} & 0 & \vdots & 0 \\
\vdots & 0 & Z_{3_{L}} & 0 & \vdots \\
0 & \vdots & 0 & \ddots & 0 \\
0 & 0 & \cdots & 0 & Z_{n_{L}}
\end{array}\right] .
$$

The load matrix that is separable from the impedance matrix is an advantage as individual parasitic element's load can be altered separately, which saves computation.

From (2), the current $\mathbf{I}$ is a function of the load matrix $\mathbf{Z}_{\mathbf{L}}$, and the radiation intensity for a given direction is a function of the current $\mathbf{I}$ in turn as in (1) [7]. Once the current excitations are known, the SPA antennas can be seen working as phased array. Thus, the beam direction is controlled by adjusting the current excitations (amplitudes and phases) in the parasitic elements [6].

\section{COMBINATIONAL PARASITIC LOADING METHOD (CPLM)}

This work assumes the use of an ideal On/Off RF switch for toggling the parasitic elements between the short and opencircuited states. For increased azimuth beam steering resolution, a combinational approach of the parasitic impedance loads is presented.

The presented beam steering technique makes use of the combination of the possible switching states of the impedance load connected to the parasitic element and the number of parasitic elements in the array. Thus, the possible combinations or parasitic loading configurations to test are given by the combination of the number of the states that the impedance load $\left(L_{s}\right)$ can attain together with the number of parasitic elements $(P)$ :

$$
\text { Total Combinations }=\left(L_{s}\right)^{P} .
$$

If an On/Off switch is assumed and the antenna system in Fig. 1 is modelled, $16=2^{4}$ combinations or parasitic loading configurations can be tested [19].

\section{A. CPLM selection criteria}

Different loading configurations could yield different or similar results due to the symmetry property of the circular
SPA antennas. Valuable configurations may be selected based on the several criteria, for instance:

- For relative directional radiation patterns, a beam should have a minimal possibility of interference in other directions, which means a well-shaped pattern with a single lobe with maximum gain and possibly low side lobe level (SLL), is preferred;

- A configuration resulting in such a beam should also yield an array gain $G \approx 5 \mathrm{dBi} .5 \mathrm{~dB}$ gain is chosen to match most 5-Elements circular SPA antenna geometries in the literature, which attain more than $5 \mathrm{~dB}$;

- The magnitude of scattering parameter $\left|S_{1,1}\right|<-10 \mathrm{~d} B$, i.e. return loss $R L>10 d B$. Return loss of $10 \mathrm{~dB}$ is normally the minimal requirement for an antenna to have a better performance [4]; and

- $\quad$ Magnitude of scattering parameter $\left|S_{1,1}\right|<-10 \mathrm{~dB}$, return loss $R L>10 \mathrm{~dB}$.

\section{METHOD TESTING}

The proposed method was tested on three platforms, namely MATLAB version 7.4.0.287 (R2007a), WIPL-D [24], and antenna measurements in an anechoic chamber.

Most tests were performed using the MATLAB tool. For all simulations based on MATLAB, we assumed the use of the Induced EMF method to compute antenna impedances. The WIPL-D simulations were based on the Method of Moments (MoM).

\section{A. MATLAB Simulation}

The procedure for testing the Combinational Parasitic Loading Method using the MATLAB tool is shown is Fig. 2. An initial fixed geometry was generated using the MATLAB tool. Thereafter, the impedance matrix which consists of the self and mutual impedances was computed based on the induced EMF method. The currents for the individual elements were then solved using (2). The initial impedance matrix was used as a reference throughout the simulations, because only the load matrix was changed when the parasitic impedance loads were altered.

The procedure outlined in Fig. 2 was followed until all the loading configurations have been tested. Testing of every loading configuration was performed following the proposed method selection criteria, which are discussed above.

\section{B. WIPL-D simulation}

WIPL-D Pro 3D EM Solver Lite v6.0 is a commercial software package for high frequency electromagnetic modeling and simulation [24]. Simulation in WIPL-D followed almost the same procedure as indicated in Fig. 2 for simulations in MATLAB. But WIPL-D used the method of moments to solve the impedance matrix. In addition, only the initial configuration was defined in WIPL-D because most functions employed in Fig. 2 are built in. However, the selection criteria in WIPL-D were performed manually (perhaps by even incorporating WIPL-D and MATLAB) by comparing the results to the selection requirements. 


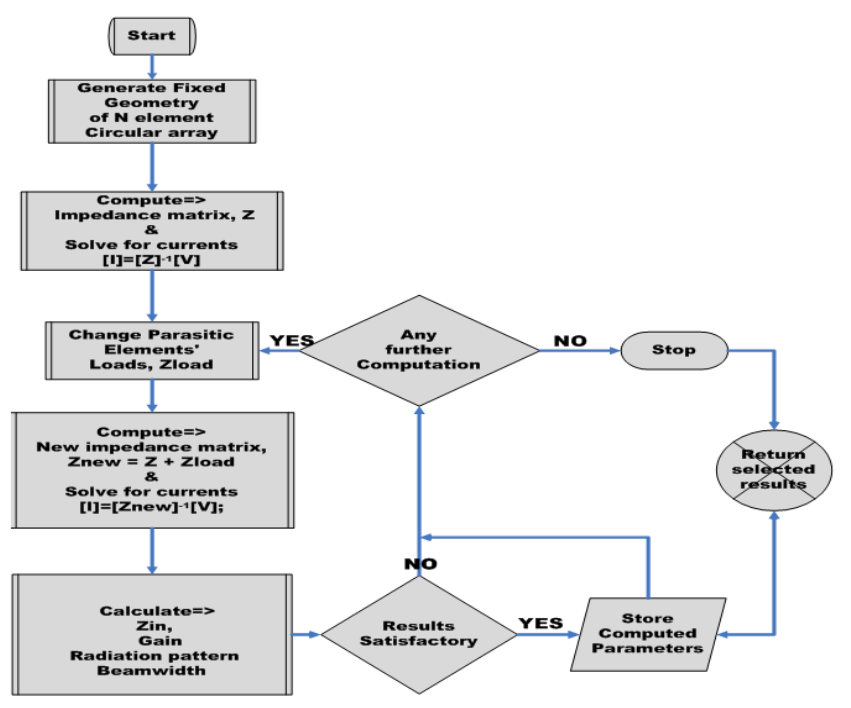

Fig. 2: Flow chart for testing combinational parasitic loading method

\section{Antenna measurements}

To verify numerical results, the combinational parasitic loading method was tested on a prototyped SPA antenna. The radiation pattern measurements were carried out in the anechoic chamber [22], where the Scientific Atlanta Compact Range measurement facilities were used. Only a subset of the total simulation results was tested in this regard because most of the results were expected to be the same due to the SPA antenna's symmetry. The radiation patterns and maximum gain were measured; the input impedance values were only simulated. The SPA prototype was made of the monopole elements and a large ground plane (which is much greater than the wavelength at $2.4 \mathrm{GHz}$ ). The SPA prototype's specifications were derived from those of the dipole array shown in Fig. 1, with the following additions:

- Dimensions of ground plane

○ Thickness, $t=3 \mathrm{~mm}$;

○ Surface area, Lenght $\times$ Width $=300 \mathrm{~mm} \times 300 \mathrm{~mm}$; and

- SubMiniature version A (SMA) connector $50 \Omega$; used for feeding the active element.

Although we assumed the use of an ideal On/Off RF switch in this work, the open-circuited parasitic elements were isolated from the ground plane by using an insulation material made of a paper and "Bostik Prestik" [25]. This insulation material might be introducing some capacitance between the ground plane and the parasitic elements. However, the capacitance is assumed to be negligibly small and hence not accounted for. The short-circuited parasitic elements are directly shorted to the ground plane without the use of any RF switch.

\section{RESULTS AND DISCUSSIONS}

The complete set of results from the MATLAB and WIPL-D simulations and measurements are presented herein. Table 1 summarizes the results of the SPA antenna gain, input impedance and return loss for all combinations of the parasitic elements' impedance loads. Considering the circular SPA antenna's symmetry, these results can be grouped in to six unique configuration categories:

- All parasitic elements open-circuited (All-OC). The corresponding combination: $\#=\{1\}$;

- One parasitic element open-circuited and the rest shorted (1OC). The corresponding set of combinations: $\#=\{8,12,14,15\}$;

- Two neighbouring parasitic elements open-circuited and the other two shorted $(2 \mathrm{~N}-\mathrm{OC})$. The corresponding set of combinations: $\#=\{4,7,10,13\}$;

- Two opposite parasitic elements open-circuited and the other two shorted $(2 \mathrm{Op}-\mathrm{OC})$. The corresponding set of combinations: $\#=\{6,11\}$;

- Three parasitic elements open-circuited and one shorted (3OC). The corresponding set of combinations: $\#=\{2,3,5,9\}$; and

- All the parasitic elements short-circuited (All-SC). The corresponding combination: $\#=\{16\}$.

The Parasitic loading combinations that yield the same loading configurations produce similar antenna attributes (gain, input impedance and radiation pattern) due to the symmetry of the SPA antenna. These symmetrical properties can be noticed in Table 1 from similar loading configurations.

Fig. 4 to Fig. 6 presents some of the azimuth radiation patterns of the six major loading configuration categories. Almost all the loading configurations meet the gain and return loss selection criteria. The transmission line impedance, $\mathrm{Z} 0=$ $50 \Omega$ is assumed for testing antenna impedance match. All figures (Fig. 4 to Fig. 6) present the radiation pattern results from MATLAB, WIPL-D and antenna measurements.

The three sets of results match very well with maximum gains being at same angles although levels of side lobe vary. The minor deviations amongst the three sets of results might be due to the manufacturing errors of the prototype, different level of accuracy between the induced EMF method and the method of moments. Otherwise, all figures represent different position along the azimuth plane in which the main beam can be directed. Employing a permutation of the parasitic elements as in Table 1, would result in the radiation pattern being steered in different directions.

The loading configuration of the $2 \mathrm{~N}-\mathrm{OC}$ yields acceptable results based on the selection criteria of the radiation patterns.

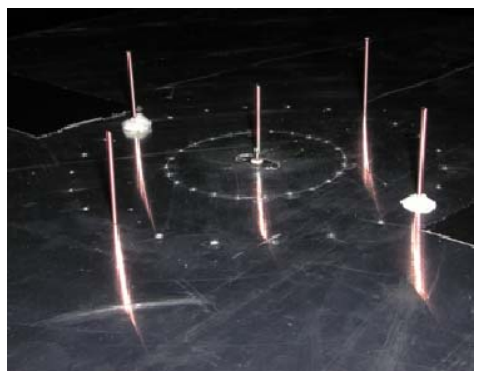

Fig. 3: Prototype of modeled switched parasitic array antenna under test. 
TABLE 1: SIMULATED ARRAY GAIN (G); INPUT IMPEDANCE ( $\mathrm{Z}_{\mathrm{IN}}$ ); RETURN LOSS (RL) CORRESPONDING TO THE PARASITIC ELEMENT'S IMPDANCE LOAD COMBINATIONS. “O” REPRESENTS OPEN-CIRCUITED AND "S" REPRESENTS SHORT-CIRCUTED PARASITIC ELEMENTS RESPECTIVELY.

\begin{tabular}{|l|l|l|l|l|l|l|l|}
\hline$\#$ & $\mathbf{P}_{\mathbf{1}}$ & $\mathbf{P}_{\mathbf{2}}$ & $\mathbf{P}_{\mathbf{3}}$ & $\mathbf{P}_{\mathbf{4}}$ & $\begin{array}{l}\mathbf{G} \\
(\mathbf{d B i})\end{array}$ & $\mathbf{Z}_{\text {in }}(\mathbf{\Omega})$ & $\begin{array}{l}\mathbf{R L} \\
(\mathbf{d B})\end{array}$ \\
\hline 1 & $O$ & $O$ & $O$ & $O$ & 3.32 & $44.24-55.29 \mathrm{i}$ & 13.52 \\
\hline 2 & $O$ & $O$ & $O$ & $S$ & 4.54 & $51.79-58.52 \mathrm{i}$ & 13.92 \\
\hline 3 & $O$ & $O$ & $S$ & $O$ & 4.54 & $51.79-58.52 \mathrm{i}$ & 13.92 \\
\hline 4 & $O$ & $O$ & $S$ & $S$ & 6.02 & $66.5-61.13 \mathrm{i}$ & 14.63 \\
\hline 5 & $O$ & $S$ & $O$ & $O$ & 4.54 & $51.79-58.52 \mathrm{i}$ & 13.92 \\
\hline 6 & $O$ & $S$ & $O$ & $S$ & 5.62 & $57.6-65.12 \mathrm{i}$ & 13.03 \\
\hline 7 & $O$ & $S$ & $S$ & $O$ & 6.02 & $66.5-61.13 \mathrm{i}$ & 14.63 \\
\hline 8 & $O$ & $S$ & $S$ & $S$ & 5.86 & $83.32-70.68 \mathrm{i}$ & 13.16 \\
\hline 9 & $S$ & $O$ & $O$ & $O$ & 4.54 & $51.79-58.52 \mathrm{i}$ & 13.92 \\
\hline 10 & $S$ & $O$ & $O$ & $S$ & 6.02 & $66.5-61.13 \mathrm{i}$ & 14.63 \\
\hline 11 & $S$ & $O$ & $S$ & $O$ & 5.62 & $57.6-65.12 \mathrm{i}$ & 13.03 \\
\hline 12 & $S$ & $O$ & $S$ & $S$ & 5.86 & $83.32-70.68 \mathrm{i}$ & 13.16 \\
\hline 13 & $S$ & $S$ & $O$ & $O$ & 6.02 & $66.5-61.13 \mathrm{i}$ & 14.63 \\
\hline 14 & $S$ & $S$ & $O$ & $S$ & 5.86 & $83.32-70.68 \mathrm{i}$ & 13.16 \\
\hline 15 & $S$ & $S$ & $S$ & $O$ & 5.86 & $83.32-70.68 \mathrm{i}$ & 13.16 \\
\hline 16 & $S$ & $S$ & $S$ & $S$ & 5.17 & $123.02-3.11 \mathrm{i}$ & 10.14 \\
\hline
\end{tabular}

From Table 1, the $2 \mathrm{~N}-\mathrm{OC}$ loading configuration has the highest gain and best return loss when compared to all other loading configurations. In addition, the $2 \mathrm{~N}-\mathrm{OC}$ loading configuration produces a directive radiation pattern as indicated in Fig. 4.

Fig. 4 shows the radiation patterns with the main beam (having maximum gain) directed at the azimuth angle $315^{\circ}$. These radiation patterns are for the $2 \mathrm{~N}-\mathrm{OC}$ loading configuration with parasitic elements at $0^{\circ}$ and $270^{\circ}$ being opencircuited. The main beam is directed towards that angle intermediate between the two open-circuited parasitic elements. This configuration allows beam steering in four discrete angles. The main beam can be pointed in these azimuth directions: $\phi_{A m}=\left\{45^{0}, 135^{\circ}, 225^{\circ}, 315^{\circ}\right\}$. To direct the beam in other angles in the azimuth plane, $\phi_{A m}$ other than $315^{\circ}$, a permutation of the $2 \mathrm{~N}-\mathrm{OC}$ loading configuration is performed around the circle.

The other loading configurations such as the $2 O p-O C$ and 3-OC yield reasonable SPA antenna gain and acceptable return loss, but do not produce the radiation patterns that meet the selection criteria of directional patterns. In Fig. 5, the azimuth radiation patterns for the loading configuration of one isolated parasitic element $((1 O C))$ at $90^{\circ}$ and three short-circuited parasitic elements at $0^{\circ}, 180^{\circ}$ and $270^{\circ}$ is presented. The main beam was expected to be pointing in the direction of the opencircuited parasitic element positioned at $90^{\circ}$ [4].

However, Fig. 5 shows a slight deviation in the main beam positioning. The main beam is slit in to the lobes having maximum gain at $40^{\circ}$ and $140^{\circ}$. We believe this inaccuracy in the main beam positioning is because of the SPA antenna structural parameters that are estimated (not optimized) in this work. Therefore, there might be a need to optimize the

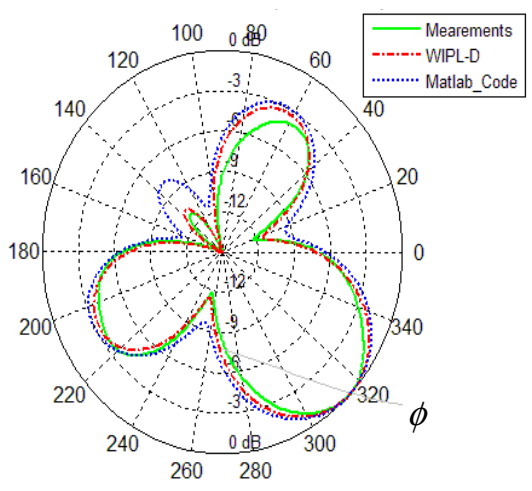

Fig. 4: Azimuth patterns for a configuration two isolated neighboring parasitic elements at $0^{\circ}$ and $270^{\circ}$ and two short-circuited parasitic elements at $90^{\circ}$ and $180^{\circ} ;(2 \mathrm{~N}-\mathrm{OC})$.

structural parameters to achieve proper beam pointing for the (1OC) loading configuration.

For the loading configuration of the two open-circuited parasitic elements $(2 \mathrm{Op}-\mathrm{OC})$, the beam is directed towards the two open-circuited elements. In this case, the radiation pattern has more than one radiation pattern lobe with maximum gain. This kind of radiation pattern does not meet the selection criteria

The 3-OC loading configurations produces the radiation patterns that are slightly different from the ones of the 1-OC loading configuration in terms of the side lobe levels (SLL) and pattern directionality. Other than that, the 1-OC and 3-OC loading configurations produce similar radiation patterns. Nonetheless, the antenna and return loss of the two configurations differ arbitrarily as can be seen from Table 1. The $1-O C$ loading configuration pattern can be preferable over the 3-OC loading configuration due to several nulls in the 1-OC loading configuration pattern. The last two configurations of All-OC and All-SC do not yield a directional pattern. The two configurations could be used when the SPA antenna is being configured for an omnidirectional pattern. Between, the two configurations, the All-OC loading configuration yields a better omnidirectional pattern resemblance. Fig. 6 presents the azimuth radiation patterns produced by the All-OC loading configurations.

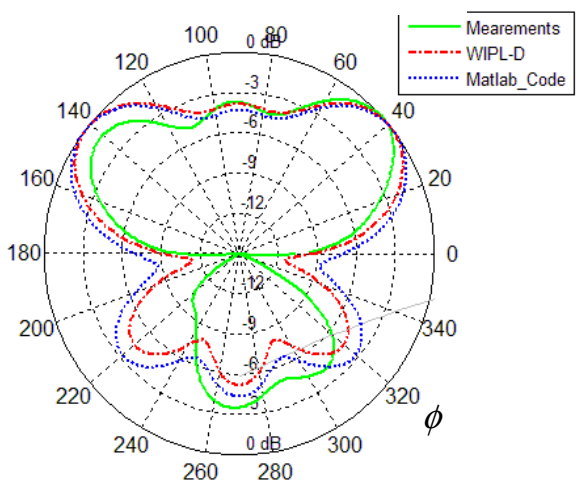

Fig. 5: Azimuth patterns for a configuration of one isolated parasitic element at $90^{\circ}$ and 3 short-circuited parasitic elements at $0^{\circ}, 180^{\circ}$ and $270^{\circ}$; (1-OC). 


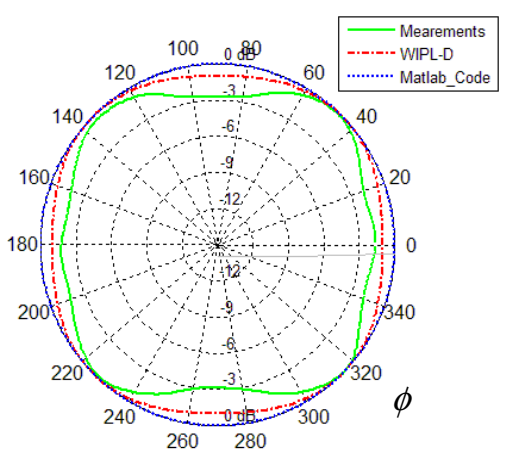

Fig. 6: Azimuths pattern for a configuration of all parasitic elements opencircuited at $0^{\circ}, 90^{\circ}, 180^{\circ}$ and $270^{\circ}$; (All-OC).

\section{CONCLUSION AND FUTURE WORK}

A conventional beam steering method for the circular SPA antennas is achieved by open-circuiting one parasitic element at a time. This method results in a limited beam steering resolution for the SPA antennas with few parasitic elements. To enhance the beam steering resolution for the circular SPA antennas, a beam steering technique is proposed. The proposed method is tested on the circular SPA antenna design without optimized structural parameters. Therefore, only a limited set of results have directional radiation patterns with a single define direction of the main beam.

Despite some radiation patterns having more than one lobe with maximum gain, the proposed method has introduced several additional loading configurations, while still using the common circular SPA antenna geometry. These additional loading configurations can increase the azimuth beam steering resolution of the circular SPA antennas, as compared to the conventional beam steering method. The results from the two simulations and the antenna measurements match very well. Minor deviations in the radiation patterns of the three sets of results are attributed to the inaccuracies in the testing platform(s).

Further work can still be done to test the proposed beam steering method on the SPA antenna geometry, designed with optimized structural parameters. Also, practical measurements of the input impedance can be performed to compare with the simulation results.

\section{ACKNOWLEDGMENTS}

We would like to thank the members of the staff of the Electromagnetic Centre of the University of Pretoria [26], namely Professor Wimpie Odendaal and Mr. Lukas Naude who helped us with the process of antenna measurements.

\section{REFERENCES}

[1] A. Gkelias and K. Leung, "Multiple Antenna Techniques for Wireless Mesh Networks," Wireless Mesh Networks, pp. 277-307, 2007.

[2] F. Babich, M. Comisso, and L. Mania, "Multi-Antenna Techniques for Wireless Mesh Networks in an Outdoor Environment," in Communications, 2007. ICC '07. IEEE International Conference on, 2007, pp. 4961-4966.

[3] J. Stine, "Exploiting smart antennas in wireless mesh networks using contention access," Wireless Communications, IEEE, vol. 13, no. 2, pp. 38-49, 2006

[4] D. Thiel and S. Smith, Switched parasitic antennas for cellular communications. Artech House Publishers, 2001.
[5] W. Stutzman and G. Thiele, Antenna theory and design. John Wiley \& Sons, Inc., 1981

[6] B. Schaer, K. Rambabu, J. Bornemann, and R. Vahdieck, "A simple algorithm for the control of reactances in beam steering applications with parasitic elements," presented at the Wireless Communication Technology, 2003. IEEE Topical Conference on, 2004, pp. 394-395.

[7] R. Islam and R. Adve, "Beam-forming by mutual coupling effects of parasitic elements in antenna arrays," presented at the Antennas and Propagation Society International Symposium, 2002. IEEE, 2002, vol. 1, pp. 126-129.

[8] H. Scott and V. Fusco, " $360^{\circ}$ electronically controlled beam scan array," Antennas and Propagation, IEEE Transactions on, vol. 52, no. 1, pp. 333-335, 2004.

[9] M. Migliore, D. Pinchera, and F. Schettino, “A simple and robust adaptive parasitic antenna," Antennas and Propagation, IEEE Transactions on, vol. 53, no. 10, pp. 3262-3272, 2005.

[10] R. Schlub and D. Thiel, "Switched parasitic antenna on a finite ground plane with conductive sleeve," Antennas and Propagation, IEEE Transactions on, vol. 52, no. 5, pp. 1343-1347, 2004.

[11] T. Ohira and K. Iigusa, "Electronically steerable parasitic array radiator antenna," Electronics and Communications in Japan(Part II Electronics), vol. 87, no. 10, pp. 25-45, 2004.

[12] S. Panagiotou, S. Mitilineos, T. Dimousios, and C. Capsalis, "A broadband, circular switched parasitic array for portable and vehicular mobile DVB T applications at the V UHF band," Microwave and Optical Technology Letters, vol. 50, no. 7, pp. 1727-1732, 2008.

[13] S. Panagiotou, S. Mitilineos, T. Dimousios, and C. Capsalis, "A Broadband, Vertically polarized, circular switched parasitic array for indoor portable DVB-T applications at the IV UHF band," Broadcasting, IEEE Transactions on, vol. 53, no. 2, pp. 547-552, 2007.

[14] R. Milne, “A small adaptive array antenna for mobile communications," presented at the Antennas and Propagation Society International Symposium, 1985, 2002, vol. 23, pp. 797-800.

[15] M. Kamarudin and P. Hall, "Switched beam antenna array with parasitic elements," Progress In Electromagnetics Research, vol. 13, pp. 187-201, 2009.

[16] R. Harrington, "Reactively controlled directive arrays," Antennas and Propagation, IEEE Transactions on, vol. 26, no. 3, pp. 390-395, 2002.

[17] A. Sibille, C. Roblin, and G. Poncelet, "Circular switched monopole arrays for beam steering wireless communications," Electronics Letters, vol. 33, no. 7, pp. 551-552, 1997.

[18] L. Petit, L. Dussopt, and J. M. Laheurte, "MEMS-switched parasiticantenna array for radiation pattern diversity," Antennas and Propagation, IEEE Transactions on, vol. 54, no. 9, pp. 2624-2631, 2006

[19] M. Mofolo, A. Lysko, and W. Clarke, "A method of electronic beam steering for circular switched parasitic dipole arrays," presented at the Southern Africa Telecommunication Networks and Applications Conference (SATNAC), Spier Estate, Stellenbosch, South Africa, 2010, pp. 5-8.

[20] R. O. M. Mofolo, "Enhanced Beam Steering and Parameter Analyses for Switched Parasitic Arrays," submitted for the degree of Master in Electrical and Electronic Engineering Science, University of Johannesburg, 2011.

[21] S. J. Orfanidis, "Electromagnetic Waves and Antennas." [Online]. Available: http://www.ece.rutgers.edu/ orfanidi/ewa/. [Accessed: $25-$ Oct-2010].

[22] C. Balanis, Antenna theory, 3rd ed. John Wiley \& Sons, Inc., 2005.

[23] R. Schlub, "Practical realization of switched and adaptive parasitic monopole radiating structures," Ph.D. dissertation, Griffith University, 2004.

[24] WIPL-D Pro Lite v6.0 3D EM solver. WIPL-D Ltd, 2005.

[25] Bostik Ltd., "Prestik | Stationery | Products | Bostik." [Online]. Available: $\quad$ http://www.bostik.co.za/products/stationery/prestik. [Accessed: 04-Apr-2011].

[26] "Electromagnetism [Department of Electrical, Electronic and Computer Engineering]." [Online]. Available: http://www.ee.up.ac.za/main/en/research/electromagnetism. [Accessed: 11-May-2011]. 